\title{
Psychosis, Treatment Emergent Extrapyramidal Events, and Subsequent Onset of Huntington's Disease: A Case Report and Review of the Literature
}

\author{
Changqing $\mathrm{Xu}^{1}$, Jegan Yogaratnam ${ }^{1}$, Nigel $\operatorname{Tan}^{2}$, Kang $\mathrm{Sim}^{1}$ \\ Institute of Mental Health/Woodbridge Hospital, ${ }^{2}$ National Neuroscience Institute, Singapore
}

Huntington's disease (HD) is an autosomal dominant neurodegenerative disease characterized by a triad of progressive motor dysfunction, cognitive decline and psychiatric disturbances. The hallmark of HD is the distinctive choreiform movement disorder that typically has a subtle, insidious onset in the fourth to fifth decade of life and gradually worsens over 10 to 20 years until death. Notably, two-thirds of HD patients present with chorea and one third with mental changes. The prevalence of psychiatric symptoms is significantly higher than in the general population, and is estimated to be around 66-73\%. Here, we report a unique case of subsequent onset of HD in a patient previously treated for schizophrenia and complicated by the extrapyramidal side effects to antipsychotics.

KEY WORDS: Huntington's disease; Schizophrenia; Neuropsychiatric manifestations; Extrapyramidal side effects.

\section{INTRODUCTION}

Huntington's disease (HD) is an autosomal dominant neurodegenerative disease characterized by a triad of progressive motor dysfunction, cognitive decline and psychiatric disturbances. ${ }^{1-3)}$ The genetic mutation underlying this disorder has been localized to the short arm of chromosome 4, which results in an expansion and instability of a polymorphic trinucleotide base cytosine-adenine-guanine repeat (CAG repeat) in gene IT 15 producing unstable Huntingtin protein. ${ }^{4)}$ Healthy individuals have around 11-35 CAG repeats on this gene, whereas HD patients have 36 or more. ${ }^{5)}$ The hallmark of HD is the distinctive choreiform movement disorder that typically has a subtle, insidious onset in the fourth to fifth decade of life and gradually worsens over 10 to 20 years until death. ${ }^{6}$ Notably, two-thirds of HD patients present with chorea and one third with mental changes. The prevalence of psychiatric symptoms is significantly higher than in the general population, and is estimated to be around $66-73 \%{ }^{7)}$ Both neurotic and psychotic disorders have been reported in patients with HD. Psychotic symptoms occur in about

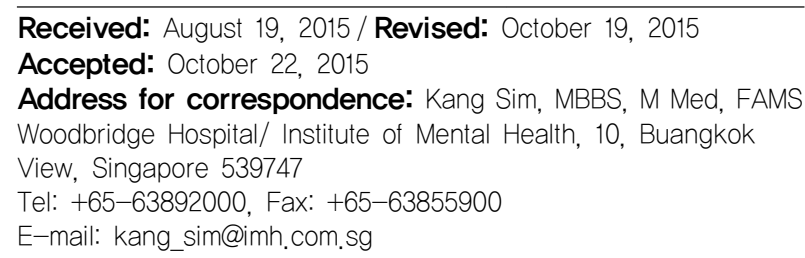

$3-11 \%$ of patients, while a more specific schizophrenialike psychosis occurs in $3-6 \%$ of patients. ${ }^{7,8)}$ Here, we report a unique case of subsequent onset of HD in a patient previously treated for schizophrenia and complicated by the extrapyramidal side effects (EPSE) to antipsychotics.

\section{CASE}

The patient is a 48-year-old Chinese female who was unemployed and divorced with two adult children. She was first presented to a local tertiary psychiatric hospital in 2001 with 4 years of untreated duration of persecutory delusions, delusion of jealousy, third person auditory hallucinations, threats to hurt herself and her two children and decline in her occupational functioning. She was diagnosed with schizophrenia and was treated with risperidone $1.5 \mathrm{mg}$ once at night. Due to her poor drug adherence, a monthly intra muscular injection of flupenthixol decanoate $20 \mathrm{mg}$ every four weekly was prescribed. In 2008, she was noted to have mild EPSE, such as tremors, cogwheel rigidity and orofacial dyskinesia. She was treated with benzhexol $2 \mathrm{mg}$ on every morning and depot flupenthixol decanoate was discontinued subsequently.

She was admitted to the inpatient psychiatric ward in 2011 (index admission) due to unusual behaviour of talking to herself irrelevantly, paranoid ideas of harm by her family resulting in violence, poor appetite, poor self-care

(c) This is an Open-Access article distributed under the terms of the Creative Commons Attribution Non-Commercial License (http://creativecommons.org/licenses/by-nc/4.0) which permits unrestricted non-commercial use, distribution, and reproduction in any medium, provided the original work is properly cited. 
and significant decline in functioning. Upon admission, she was noted to have remarkable abnormal movements. She had an unsteady broad-based gait accompanied with wide arm abduction and zigzag progression due to lurching of her trunk. Walking was interrupted with pauses, backward stepping and stamping of feet with concomitant involuntary jerky movements of the upper limbs. She was unable to stand straight despite a negative Romberg sign and exhibited chewing, extruding lip and serpentine tongue movements. Her mental state examination revealed ataxic speech, blunted affect and poor insight into her illness. Her initial mini mental state examination (MMSE) score was 11 on admission (orientation, 3/10; registration, 1/3; attention and calculation, 4/5; recall, 0/3; language, 3/9) and improved to 17 in two weeks after remission of her psychotic symptoms (orientation, 5/10; registration, 3/3; attention and calculation, 4/5; recall, 2/3; language, 3/9). Bedside cognitive testing revealed impairment in frontal lobe functioning, such as verbal fluency, set shifting and abstract thinking.

Corroborative history revealed that she first had mild 'swinging' movements of lower limbs in 2008. Over the past years prior to her index presentation, the abnormal movements gradually spread to her hands and body, and worsened for past year, which resulted in her inability to speak fluently, tendency to fall and decline in her daily functioning. Further probing highlighted that she had a strong family history of mental illness and HD. Her eldest brother was diagnosed with schizophrenia. Her second brother was diagnosed with HD and died at the age of 50 years due to intra-cranial haemorrhage following a fall. Her deceased father was suspected to have suffered from HD. In view of above clinical symptoms and positive family history of HD, DNA analysis for HD using the polymerase chain reaction analysis was performed. The results showed that the two alleles of the CAG repeats in the Huntingtin gene had 17 and 41 repeats, which confirmed the diagnosis of HD. Neuroimaging of the brain was not repeated as the brain computed tomography performed in 2004 was normal.

Her diagnosis for her index admission was revised to organic psychotic disorder secondary to HD in someone with a strong family history of HD and previous diagnosis of schizophrenia with EPSE to antipsychotics. She was treated with risperidone $2 \mathrm{mg}$ twice daily, fluoxetine 20 $\mathrm{mg}$ once in the morning, benzhexol $2 \mathrm{mg}$ once in the morning and sodium valproate controlled release (CR) $500 \mathrm{mg}$ once at night. In addition, family education was provided and her children were encouraged to undergo genetic counseling. She gradually showed improvement in her behavior with less agitation, aggression and resolution of persecutory delusion. Her current mental state at the time of this report showed she is stable with no behavioral issues in a community home facility. Her choreiform movements persist and are prominent now, verbal output is minimal and her affect is constricted.

\section{DISCUSSION}

We presented a unique case of a lady whose psychotic illness possibly predated her onset of choreiform movements by slightly more than a decade and is complicated by the onset of treatment emergent EPSE secondary to antipsychotics. Another possibility is the onset of HD following the antecedent course of schizophrenia with antipsychotic induced EPSE. Neuropsychiatric manifestations may be one of the earliest markers of HD, in addition to changes in motor functioning, striatal volume and cognitive performance. ${ }^{9)}$ Neuropsychiatric symptoms, such as psychosis, sometimes present more than 10 years before a formal diagnosis of HD. Patients with an early age of onset and family history of HD seem to have a greater risk of developing psychosis. ${ }^{1,10)}$ Of the schizophrenia-like psychoses that occur in HD disease, the paranoid form is the most common subtype. ${ }^{11)}$ Though our patient has a strong family history of HD, she probably developed HD at the usual age of onset of HD around the fourth to fifth decade of life and with the potential for inexorable HD progression over the next 1-2 decades. ${ }^{12)}$ Positive psychotic symptoms do not follow a characteristic course and tend to become less overt as the disease, including cognitive impairments progress. ${ }^{13)}$ In this patient, florid persecutory delusions and auditory hallucinations were present in her acute psychiatric presentations.

In addition to psychosis, there are other psychiatric co-morbidities such as personality changes, neurocognitive changes, depression, which can occur in up to $95 \%$ of patients with HD. ${ }^{10)}$ Most commonly, personality changes may occur including increasing suspiciousness, irritability, emotional lability, impulsivity, aggression or apathy, ${ }^{1,9,11)}$ which may suggest frontal lobe involvement. ${ }^{14)}$ Depression happens in up to $32-44 \%$ of HD patients, and can precede the more typical HD features, such as chorea and dementia by 2-20 years, and is thought to be related to early degeneration of the medial caudate. ${ }^{1,14)}$ Subsequent to diagnosis of HD, patients may develop psychiatric manifestations such as aggression, impulsivity, affective changes such as depression, suicidality, or behavioural 
features including social withdrawal. ${ }^{10)}$

Cognitive deterioration is an inevitable and progressive feature, which presents in almost all HD patients and typically begins 1 year before or after the onset of chorea ${ }^{1,10)}$ and was evident in our case. Deficits in memory, visuospatial abilities and judgement in late-stage HD can be profound, akin to patients with Alzheimer's disease. ${ }^{2)}$ Generally, the cognitive deficits of HD reflect subcortical involvement as aphasia and agnosia are usually less obvious compared with that seen in cortical dementia. ${ }^{2)}$ It is crucial to recognize cognitive deficits with concomitant behavioural changes associated with HD which may contribute to the loss of independence and resultant functional disability more significantly than the primary motor deficits in some instances. $^{10)}$

Although a confirmatory test for HD is currently available, to date, there is no specific treatment which can prevent or reverse the clinical progression of HD. ${ }^{15)}$ Extant medications tried include tetrabenazine, amantadine, remacemide, benzodiazepines, riluzole and co enzyme Q10, ${ }^{16,17)}$ but none was proven to be fully effective in extant studies. There is also little evidence to suggest efficacy of acetylcholinesterase inhibitors on cognitive functioning in HD. ${ }^{14)}$ However, symptomatic treatment of both movement and emotional disturbances can potentially improve the quality of life in these individuals with HD. ${ }^{15)}$ Atypical antipsychotics or mood stabilisers such as sodium valproate have been shown to improve psychotic and behavioural manifestations. ${ }^{15)}$ Of note, some patients may present with extra pyramidal motor abnormalities (induced by antipsychotics in our case) such as rigidity, tremors and akathisia before chorea manifests, ${ }^{18)}$ which may result in delay of the clinical diagnosis of HD especially in patients with known psychiatric disorders treated with psychotropic medications.

This report highlights that the neuropsychiatric symptoms of HD including psychotic manifestations and treatment emergent neurological side effects are complex and disabling, and may antedate the motor features by many years, as was demonstrated in this case. Prompt diagnosis, continual monitoring and careful management of both the neurological and comorbid neuropsychiatric manifestations of HD are necessary to lessen the disease burden on both the sufferer and their caregivers.

\section{REFERENCES}

1. Lembert MV. Epilepsy, Tourette's syndrome and Huntington's disease. Med Personal Subscr 2004;32:48-50.

2. Ross CA, Margolis RL. Huntington's disease. Clin Neurosci Res 2001;1:142-152.

3. Tabrizi SJ, Langbehn DR, Leavitt BR, Roos RA, Durr A, Craufurd D, et al; TRACK-HD investigators. Biological and clinical manifestations of Huntington's disease in the longitudinal TRACK-HD study: cross-sectional analysis of baseline data. Lancet Neurol 2009;8:791-801.

4. Quintanilla RA, Johnson GV. Role of mitochondrial dysfunction in the pathogenesis of Huntington's disease. Brain Res Bull 2009;80:242-247.

5. Robins Wahlin TB. To know or not to know: a review of behaviour and suicidal ideation in preclinical Huntington's disease. Patient Educ Couns 2007;65:279-287.

6. MacDonald ME, Ambrose CM, Duyao MP, Myers RH, Lin $\mathrm{C}$, Srinidhi $\mathrm{L}$, et al. A novel gene containing a trinucleotide repeat that is expanded and unstable on Huntington's disease chromosomes. The Huntington's Disease Collaborative Research Group. Cell 1993;72:971-983.

7. van Duijn E, Kingma EM, van der Mast RC. Psychopathology in verified Huntington's disease gene carriers. $J$ Neuropsychiatry Clin Neurosci 2007;19:441-448.

8. Naarding P, Kremer HP, Zitman FG. Huntington's disease: a review of the literature on prevalence and treatment of neuropsychiatric phenomena. Eur Psychiatry 2001;16:439445.

9. Duff K, Beglinger LJ, Paulsen JS. "Pre-symptomatic" Huntington's disease. Handb Clin Neurol 2008;89:589-598.

10. Jauhar S, Ritchie S. Psychiatric and behavioural manifestations of Huntington's disease. Adv Psychiatr Treat 2010;16: 168-175.

11. Rosenblatt A, Leroi I. Neuropsychiatry of Huntington's disease and other basal ganglia disorders. Psychosomatics 2000;41:24-30.

12. Corrêa BB, Xavier M, Guimarães J. Association of Huntington's disease and schizophrenia-like psychosis in a Huntington's disease pedigree. Clin Pract Epidemiol Ment Health 2006;2:1.

13. Mendez MF. Huntington's disease: update and review of neuropsychiatric aspects. Int J Psychiatry Med 1994;24: 189-208.

14. Cubo E, Shannon KM, Tracy D, Jaglin JA, Bernard BA, Wuu $\mathrm{J}$, et al. Effect of donepezil on motor and cognitive function in Huntington disease. Neurology 2006;67:12681271.

15. Nance M, Paulsen JS, Rosenblatt A, Wheelock V. A physician's guide to the management of Huntington's disease. 3rd ed. New York:Huntington's Disease Society of America;2011.

16. Walker FO. Huntington's disease. Lancet 2007;369:218-228.

17. Landwehrmeyer GB, Dubois B, de Yébenes JG, Kremer B, Gaus W, Kraus PH, et al; European Huntington's Disease Initiative Study Group. Riluzole in Huntington's disease: a 3-year, randomized controlled study. Ann Neurol 2007;62: 262-272.

18. Lishman WA. Organic psychiatry: The psychological consequences of cerebral disorder. 3rd ed. Oxford, UK:Blackwell Publishing Company;1998. 